\title{
Elasticity and Echogenicity Analysis of Agarose Phantoms Mimicking Liver Tumors
}

\author{
Bin Luo ${ }^{1}$, Ronghua Yang ${ }^{1}$, Peng Ying ${ }^{1}$, Michael Awad ${ }^{2}$, Michael Choti $^{2}$, Russell Taylor ${ }^{3}$ \\ ${ }^{1}$ Department of Biomedical Engineering, ${ }^{3}$ Engineering Research Center for Computer Integrated Surgery and System \\ Technology, Johns Hopkins University and ${ }^{2}$ Department of Surgery, Johns Hopkins Hospital, Baltimore, MD
}

\begin{abstract}
For ultrasound elastography there currently exists no large animal model of liver lesions for testing. In this study, we modeled liver lesions using agarose gel, characterizing the phantoms' elastic and echogenic properties. An equation was derived to express a phantom's Young's modulus as a function of its agarose concentration. Moreover, we altered phantoms' echogenicities by adding various amounts of graphite, and found that its inclusion up to $2 \%$ does not significantly affect elasticity. These findings can help to systematically create desired liver tumor phantoms for numerous studies.
\end{abstract}

Index Terms - Echogenicity, elasticity, liver tissue phantom

\section{INTRODUCTION}

Ultrasound (US) elastography has been proposed for in vivo characterization of the mechanical properties of soft tissues, providing ways of cancer diagnosis and monitoring of surgical procedures [1]. Elasticity and echogenicity data on target lesions are required for US elastography, although to date there is no good model for liver lesions in large animals. This study looks at modeling liver tumors having different elasticities and echogenicities. Agarose gel phantoms have been chosen for various elastography and targeting studies since its mechanical properties closely mimic that of soft tissue [2]. Both are viscoelastic materials, their mechanical properties defined by the Young's modulus $(E)$ and the viscous modulus [3]. A material's echogenicity can be separated into three categories: tissue appearing brighter than surrounding material is referred to as hyperechoic, that appearing the same as the surrounding is isoechoic, and that appearing darker is hypoechoic. A systematic characterization of these parameters can produce guidelines to simplify creation of desired phantoms and establish a standard for reporting US elastography studies.

\section{METHODS}

\section{A. Phantom Construction}

Four series of agarose phantoms were produced. Triplicates of test samples were made for repeated testing.

The first study was conducted to determine the effect of agarose concentration on elasticity. For these experiments, agarose powder (Type I-A Low EEO, Sigma Chemical, Inc.) was mixed in water, heated until almost boiling, and then poured into cylindrical heat-resistant plastic molds. Samples were left to cool to room temperature, allowing the material to solidify. Each sample was $1.5 \mathrm{~cm}$ in height and $2.5 \mathrm{~cm}$ in diameter. The agarose concentrations included were $0.5,1.0$, $1.5,2.0,2.5,3.0,5.0$, and $10.0 \%$. The $0.5 \%$ phantoms did not solidify sufficiently and the $10 \%$ phantoms solidified with large air bubbles. They were excluded from further studies.

A second sample set was made for the echogenicity study. Graphite powder (Tube-O-Lube, Dynamic Industrial Group) was introduced into $2 \%$ agarose phantom during preparation. Nine triplicates with $0,0.05,0.1,0.15,0.2,0.5,1.0,1.5$, and $2.0 \%$ graphite concentrations were created to mimic varied echogenicity. These cylindrical phantoms measured $1.5 \mathrm{~cm}$ in height and $1.0 \mathrm{~cm}$ in diameter.

A third set duplicated the concentrations used in the second set in $2.5 \mathrm{~cm}$ diameter. Elasticity tests were performed on this set to validate the hypothesis that adding graphite does not change the phantoms' elasticity established by the first set.

The fourth set of phantoms was created to mimic isoechoic lesions with varied elasticities. $0.1 \%$ graphite was mixed in varying concentrations of agarose. These are ideal for elastography studies that seek to quantify elastic parameters of tumor tissue that are hard to detect by visualization in US.

\section{B. Elasticity}

In order to characterize elasticity of agarose phantoms, a rheometer (EnduraTEC ELF 3200 Series, BOSE) was employed to conduct mechanical compression tests. All samples were tested within 15 minutes of complete solidification to minimize alterations in mechanical properties due to dehydration. The phantoms were inserted between the plates of the rheometer and tested by cycles of 10 compressions of $1 \mathrm{~mm}$ at $1 \mathrm{~Hz}$. The rheometer measured the reciprocated force $(F)$ from compression and the depression of phantom height $(L)$. Time, $L$, and $F$ were recorded at $2000 \mathrm{~Hz}$ and stored in a text file. The data was processed by a custom MatLab program that calculated the averaged $\Delta L$ and $\Delta F$ of compression from reconstructed data. Using the sample's initial height $\left(L_{0}\right)$ and area $\left(A_{o}\right), E$ was calculated from its definition:

$$
E=\frac{\text { stress }}{\text { strain }}=\frac{\Delta F / A_{0}}{\Delta L / L_{0}}=\frac{\Delta F L_{0}}{A_{0} \Delta L} .
$$

The program allowed for quick and accurate processing. Questionable data was hand checked via Excel. 


\section{Echogenicity}

The small dimension phantoms set of $2 \%$ agarose and nine different graphite concentrations were inserted into fresh ex vivo porcine liver to allow assessment relative to a clinically relevant surrounding. Samples were placed at approximately $1 \mathrm{~cm}$ depth. Images were obtained using an Aloka SSD -1400 US machine with a $4-8 \mathrm{MHz}$ linear T-probe in cross-section to the phantom. The images were printed, digitized, and then analyzed using the ImageJ software package. All images were scanned at 600dpi with 8-bit grayscale using an Epson 3200 scanner to ensure minimum loss of resolution and color information. The average intensity of the target lesion $\left(I_{t}\right)$ and the average intensity of the background liver tissue $\left(I_{b}\right)$ were acquired to find the local contrast $(C)$, defined by

$$
C=\left(I_{t}-I_{b}\right) / I_{b} \text {. }
$$

A local contrast near zero indicates that the target is isoechoic. Positive and negative local contrast values indicate hyperechoic and hypoechoic targets respectively.

For each image, 3 regions of interest (ROI) were selected inside the lesion phantom and 3 ROI were selected from the background tissue. These tests were averaged to obtain the mean and standard deviation for the local contrast.

\section{Elasticity And Echogenicity}

The phantoms in the $2.5 \mathrm{~cm}$ diameter set of $2 \%$ agarose and nine different graphite concentrations were put into the rheometer for compression tests. The exact testing and processing procedures described in part B were followed. Isoechoic $0.1 \%$ graphite phantoms with varying elasticities were examined using the methods described in part $\mathrm{C}$.

\section{RESULTS}

Fig. 1 plots $E$ against agarose concentration. Linear regression test performed on the data gathered from the range of $1-5 \%$ agarose gave, with $r^{2}=0.9534$, the equation

$$
\mathrm{E}=1.23 \text { [agarose]-1.245. }
$$

This linear equation can guide creation of phantoms of specific $E$ s desired in studies that look at elasticity.

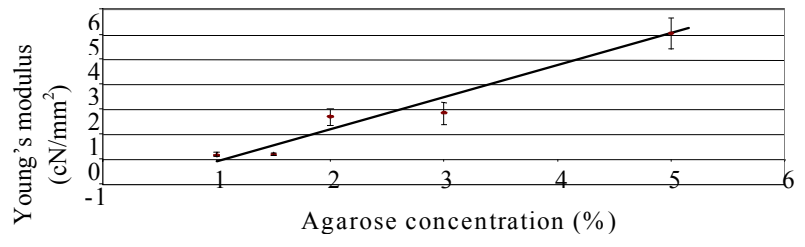

Fig. 1. Young's modulus as a function of agarose concentration.

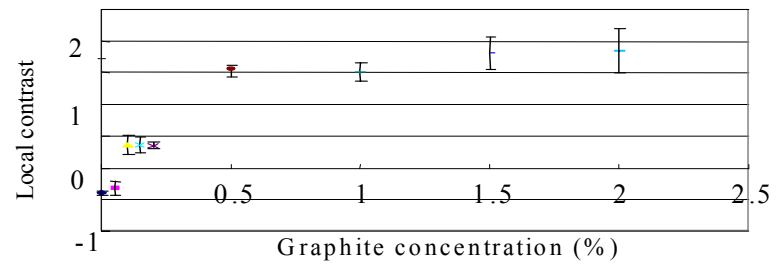

Fig. 2. Local contrast as a function of graphite concentration.

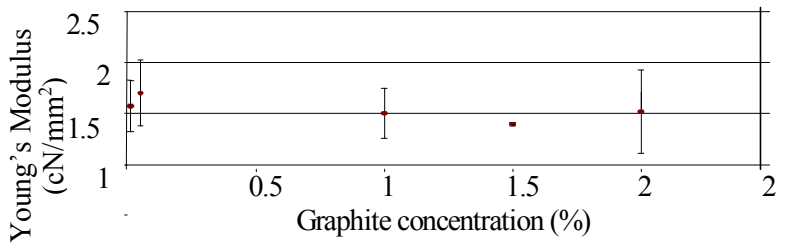

Fig. 3. Young's modulus of $2 \%$ agarose does not change significantly with variation in graphite concentration

The second part of the study characterized the echogenicity of phantoms inside cadaveric porcine liver. Fig. 2 shows that a pure agarose phantom containing no graphite was hypoechoic, having a local contrast below 0 . Phantoms containing 0.05 to $0.1 \%$ graphite were roughly isoechoic, while those with higher concentrations of graphite showed up as hyperechoic. Graphite addition was also found to not significantly affect elasticity, as shown in Fig. 3.

\section{DISCUSSION}

By integrating the fact that small amounts of graphite does not significantly affect elasticity, phantoms of varying elasticity and echogenicity can be made according to Fig. 1 and 2. In addition to elasticity, viscoelastic materials have a viscosity component that can be tested with shear stress. Study of viscosity may lead to more realistic phantoms, but it is negligible in current strain imaging techniques.

The echogenicity test showed that all three echogenicity ranges can be produced by mixing $0-0.05 \%$ graphite into agarose. Testing of additional graphite concentrations in this range would offer a better echogenicity profile since small changes in graphite concentration produced significant changes in local contrast in this range. Further addition of graphite above this range resulted in no significant increase in local contrast and is therefore of minimal practical value.

\section{CONCLUSION}

The protocols developed in this study allow for creation of phantoms of varied elastic and echogenic properties. The current single gel construction, however, fails to offer the complex motion found in human tissues, which is the basis for advanced strain imaging analysis. Further studies can aim to develop clinically specific tumor phantoms that take pathophysiology into consideration. An example is to emulate the many moduli associated with extensive fibrosis found in cirrhosis by enclosing multiple phantoms together. Additional studies can also look at incorporating synthetic polymers that more closely mimicking the non-homogeneous nature of pathological human tissues than agarose gel.

\section{REFERENCES}

[1] E. Boctor et al, "Robotic strain imaging for monitoring thermal ablation of liver," Proc MICCAI, vol. 2, pp. 81-88, 2004

[2] M.D. Mitchell, H.1. Kundel, L. Axe, P.M. Joseph, "Agarose as a tissue equivalent phantom material for NMR imaging," Magn Reson Imag, vol. 4, pp. 263-266, 1986.

[3] D.S. Jones, "Dynamic mechanical analysis of polymeric systems of pharmaceutical and biomedical significance," Int J Pharm, vol. 179, pp 167-178, 1999. 